\title{
Insights into Broad-Specificity Starch Modification from the Crystal Structure of Limosilactobacillus Reuteri NCC 2613 4,6- $\alpha$ - Glucanotransferase GtfB
}

Tjaard Pijning,* Joana Gangoiti, Evelien M. te Poele, Tim Börner, and Lubbert Dijkhuizen

Cite This: J. Agric. Food Chem. 2021, 69, 13235-13245

Read Online

ACCESS | 亗 Metrics \& More | 回 Article Recommendations | SI Supporting Information

ABSTRACT: GtfB-type $\alpha$-glucanotransferase enzymes from glycoside hydrolase family 70 (GH70) convert starch substrates into $\alpha$ glucans that are of interest as food ingredients with a low glycemic index. Characterization of several GtfBs showed that they differ in product- and substrate specificity, especially with regard to branching, but structural information is limited to a single GtfB, preferring mostly linear starches and featuring a tunneled binding groove. Here, we present the second crystal structure of a 4,6- $\alpha$ glucanotransferase (Limosilactobacillus reuteri NCC 2613) and an improved homology model of a 4,3- $\alpha$-glucanotransferase GtfB ( $L$. fermentum NCC 2970) and show that they are able to convert both linear and branched starch substrates. Compared to the previously described GtfB structure, these two enzymes feature a much more open binding groove, reminiscent of and evolutionary closer to starch-converting GH13 $\alpha$-amylases. Sequence analysis of 287 putative GtfBs suggests that only $20 \%$ of them are similarly "open" and thus suitable as broad-specificity starch-converting enzymes.

KEYWORDS: lactic acid bacteria, GtfB, $\alpha$-glucanotransferase, glycoside hydrolase family 70 , slowly digestible $\alpha$-glucans

\section{INTRODUCTION}

Lactic acid bacteria (LAB) are known for their extracellular "coats", largely containing $\alpha$-glucan-type polysaccharides (e.g., dextran, mutan, and reuteran), which are synthesized from sucrose by glucansucrase (GS) enzymes belonging to glycoside hydrolase family 70 (GH70) (http://www.cazy.org ${ }^{1}$ ). Recently however, within GH70, $\alpha$-glucanotransferase (GT) enzyme subfamilies were discovered in $\mathrm{LAB}$ and non-LAB that are inactive on sucrose, synthesizing $\alpha$-glucans from starch-like substrates. To date, only a few GT enzymes have been biochemically characterized; ${ }^{2-12}$ most of them display $\alpha-4,6$ transglycosylation specificity, i.e., cleavage of the substrate $\alpha$ 1,4 glycosidic bond (donor half-reaction) followed by formation of an $\alpha-1,6$ glycosidic bond (acceptor half-reaction). Three subfamilies have been described, GtfB, GtfC, and GtfD, differing in bacterial origin, reaction and product specificity, and (predicted) domain organization and displaying variations of key amino acid residues in GH70 homology motifs I-IV. ${ }^{13}$ These subfamilies also provided important insights into the evolutionary relationships between GH13 $\alpha$-amylases, which also act on starch substrates, ${ }^{14}$ and the GH70 glucansucrases (acting on sucrose only), placing the GtfB, -C, and -D subfamilies as structural and functional intermediates. ${ }^{2,4,7,15}$ Notably, starch-degrading GH13 enzymes feature an opensubstrate binding groove, and we previously hypothesized that the evolution of reaction specificity from $\alpha$-amylases toward GT and GS enzymes was accompanied by structural changes involving loop architecture around the active site. ${ }^{16}$

The GtfB-type $\alpha$-glucanotransferases, exclusively found in $\mathrm{LAB}$, share $45-50 \%$ sequence identity with GSs. Several GtfB enzymes have been characterized biochemically, ${ }^{3,6,7,9-12,17}$ revealing different substrate and product specificities. For example, the well-characterized GtfB from Limosilactobacillus reuteri 121 ( $\mathrm{Lr} 121 \mathrm{GtfB})$ preferably converts linear starch-type substrates, e.g., from amylose, it synthesizes linear isomalto-/ malto-polysaccharides (IMMP), ${ }^{16-19}$ but is hardly active on amylopectin. On the other hand, for two other GtfB enzymes, identified on the basis of amino acid variations in homology motifs II and/or IV, initial characterization suggested that they act on linear (amylose) as well as branched (amylopectin) starch substrates. From amylose, the GtfB from L. reuteri NCC 2613 (Lr2613 GtfB) synthesizes a branched reuteran-type $\alpha$ glucan, ${ }^{6}$ while the GtfB from L. fermentum NCC 2970 (Lf2970 $\mathrm{GtfB})$ remarkably displays a unique $\alpha-4,3$ transglycosylation specificity. ${ }^{7}$ The activity of these enzymes on branched substrates has not been studied in detail, while such knowledge is highly relevant for the application of these enzymes for whole starch modification. For example, the GtfB from Streptococcus thermophilus was found to be suitable for converting wheat starch and potato starch. ${ }^{11,12}$ Together, the different reaction specificities of GTs offer great opportunities to convert starches to different $\alpha$-glucans, in particular, because introduction of $\alpha-1,3$ and $\alpha-1,6$ linkages decreases their digestibility in the upper human gastrointestinal tract. This property makes these $\alpha$-glucans attractive as low-glycemic ingredients in food applications. ${ }^{20-23}$

Received: September 13, 2021

Revised: October 15, 2021

Accepted: October 20, 2021

Published: October 28, 2021 
In order to exploit the varying specificities of GTs for $\alpha$ glucan synthesis using different starch substrates, understanding the GT structure-function relationship is of great value. The accessibility of the substrate binding groove and the architecture of acceptor substrate binding subsites are key factors in determining substrate preference as well as the efficiency and specificity (e.g., $\alpha-4,6$ vs $\alpha-4,3$ ) of the transglycosylation reaction. In this regard, the so far only available experimental 3D structure of a GH70 GT enzyme, a truncated $(\Delta \mathrm{N} \Delta \mathrm{V}) \mathrm{GtfB}$ from $L$. reuteri 121 (Lr121 GtfB), provided important initial insights. In its groove, two long loops (loops A1 and B) cover donor subsites -2 and -3 , leading to the hypothesis that the resulting tunnel-like feature is the main determinant of the enzyme's limited ability to process branched starch substrates. ${ }^{16}$ Interestingly, sequence alignments showed that the lengths of the A1 and B loops differ in characterized GT enzymes. ${ }^{6,16,21}$ For example, both in Lr2613 GtfB and Lf2970 GtfB these loops are much shorter; homology models of these enzymes thus predicted a much more open binding groove, ${ }^{6,7}$ a feature that may determine higher ability to process branched substrates. However, how representative these differences are within the GtfB subfamily so far has not been investigated. In addition to the loops, variations also occur in homology motifs II and IV; in GH70 glucansucrases, the structures of complexes with sucrose, maltose, or acarbose revealed residues in these motifs that interact with the donor substrate, acceptor substrate, or inhibitor, respectively. ${ }^{24,25}$ Mutational studies confirmed the contribution of these residues to reaction specificity. ${ }^{13,26}$ However, for GH70 GTs, no acceptor substrate complexes are available yet, and residues that may affect reaction specificity in these enzymes could only be identified by homology with GSs.

Deepening our understanding of GT specificity, we describe here the second crystal structure of a GH70 $\alpha-4,6-\mathrm{GT}$ from $L$. reuteri NCC 2613 (Lr2613 GtfB), along with an improved homology model of the $\alpha-4,3-\mathrm{GT}$ from $L$. fermentum NCC 2970 (Lf2970 GtfB). Both GtfB enzymes feature shorter loops $\mathrm{A} 1$ and $\mathrm{B}$ and consequently, instead of having a tunnel, feature a much more open binding groove compared to Lr121 GtfB; this allowed us to model the binding of branched starch-like substrates. Analysis of the products synthesized from starch substrates with various amounts of branching (amylose and amylopectin) revealed the importance of difference in loop architecture, in particular of loops A1 and B, of GtfB-type $\alpha$ glucanotransferases for their substrate and product specificities. Sequence analysis of 287 putative GtfB enzymes revealed an almost bimodal distribution of loop lengths, with the majority of GtfBs (e.g., Lr121 GtfB) having long A1 and B loops, while Lr2613 GtfB represents a minority with much shorter loops, and Lf2970 GtfB has intermediate loop lengths. The structure of Lr2613 GtfB in complex with acarbose, occupying acceptor substrate subsites $(+1$ to +3$)$, allowed the identification of residues that likely contribute to acceptor binding. Interestingly, the type of residue at these specific positions correlated with the length of loops $\mathrm{A} 1$ and $\mathrm{B}$. Together, our results suggest that GH70 GtfB enzymes with a more open architecture are evolutionary closer to starch-degrading GH13 $\alpha$-amylases, while those with a tunneled binding groove have evolved away. This extends our structural knowledge of GH70 GT enzymes regarding their reaction specificity and may accelerate the discovery and application of these enzymes for starch modification aimed at the synthesis of $\alpha$-glucan food ingredients (with a low glycemic index).

\section{MATERIALS AND METHODS}

Sequence Analysis. A BLAST search was performed in the nonredundant protein sequences database, using the sequence of $\operatorname{Lr} 2613$ GtfB (GenBank ASA47879.1). The resulting hits were aligned using MUSCLE (default parameters) within Jalview $2 .{ }^{27}$ The length of loop A2 (corresponding to residues 1086-1096 in Lr2613 GtfB) was used as a criterion to remove glucansucrases from the results, which have a longer loop A2 blocking donor subsites beyond $-1 .^{16,28}$ Sequences not containing a full GH70 core (domains $\mathrm{A}+\mathrm{B}+\mathrm{C}$ ) were also omitted. The final set of putative GtfB sequences was numbered (1287 ), and the variation in length of loops A1 and B was determined. Loop A1 was defined as the segment between the two $\alpha$-helices that form the subdomain between $\beta$-strand 4 and $\alpha$-helix 5 of the $(\beta \alpha)_{8^{-}}$ barrel in Lr2613 GtfB (residues 802-811); loop B comprises the loop residues between the short $\alpha$-helix and first $\beta$-strand in domain $\mathrm{B}$ (residues 590-593). In addition to these two loops, the sequence conservation of six residues (Y632, L635, H683, Y719, R720, and N792 in Lr2613 GtfB) near acceptor subsites was analyzed, using the WebLogo server ${ }^{29}$ for visualization. Furthermore, a phylogenetic tree was constructed for the 287 sequences using MEGA X, ${ }^{30}$ the maximum likelihood method based on the JTT matrix model, with partial deletion of positions containing gaps and missing data; ${ }^{31}$ the bootstrap consensus tree was inferred from 1000 bootstrap replicates. ${ }^{32}$ Each entry was annotated with the combined number of residues in loops $\mathrm{A} 1$ and $\mathrm{B}$.

Expression and Purification. For the N-terminally truncated $L$. reuteri NCC 2613 GtfB 4,6- $\alpha$-GTase protein, plasmid pET15b/gtfB$\Delta \mathrm{N}$, constructed in a previous study and encoding amino acids $417-$ 1281 of the protein, fused with an N-terminal $\mathrm{His}_{6}$-tag cleavable by a 3C protease, was used for recombinant gene expression ${ }^{6}$ using Escherichia coli BL21 star (DE3) as a host. An overnight culture of E. coli BL21 Star (DE3) containing the relevant plasmid was diluted to $1 / 100$ in Luria broth with $100 \mu \mathrm{g} \mathrm{mL}^{-1}$ ampicillin and propagated to A600 nm 0.4-0.6. Gene expression was induced by adding isopropyl$\beta$-D-thiogalactopyranoside (IPTG) at a final concentration of 0.1 $\mathrm{mM}$, and cultivation was continued at $16{ }^{\circ} \mathrm{C}$ for $20 \mathrm{~h}$. Cells were harvested by centrifugation $(10,000 \mathrm{~g} \times 20 \mathrm{~min})$ and then disrupted with a B-PER lysis reagent (Thermo Scientific, Pierce). Following centrifugation $(15,000 \mathrm{~g} \times 20 \mathrm{~min})$, the clear supernatants were subjected to Ni-IMAC chromatography (Sigma-Aldrich). After washing the column with $25 \mathrm{mM}$ Tris- $\mathrm{HCl}(\mathrm{pH} \mathrm{8.0)}$ and $1 \mathrm{mM}$ $\mathrm{CaCl}_{2}$, bound proteins were eluted with $200 \mathrm{mM}$ imidazole in the same buffer and the imidazole was removed by use of a stirred ultrafiltration unit (Amicon, Beverly, MA) with a 30,000 molecular mass cutoff. For further purification, the protein was subjected to anion exchange chromatography on a Resource $\mathrm{Q}$ column (GE Healthcare) using $25 \mathrm{mM} \mathrm{HAc} / \mathrm{NaAc} \mathrm{pH} 5.0,150 \mathrm{mM} \mathrm{NaCl}$, and 1 $\mathrm{mM} \mathrm{CaCl}$ with a $0-1 \mathrm{M} \mathrm{NaCl}$ gradient. Purity and homogeneity were analyzed by SDS-PAGE and dynamic light scattering (DynaPro Nanostar, Wyatt Technology, Santa Barbara, USA); the protein concentration was determined using a Nanodrop 2000 spectrophotometer (Isogen Life Science, De Meern, The Netherlands). Lr121 $\mathrm{GtfB}$ and Lf2970 GtfB were produced as previously described. ${ }^{7,16}$

Substrate Specificity. Amylose V from potato starch $\left(M_{\mathrm{w}} \approx 2 \times\right.$ $10^{5} \mathrm{kDa}$ ) (AVEBE, Foxhol, The Netherlands) and amylopectin from potato starch $\left(M_{\mathrm{w}} \approx 5 \times 10^{6} \mathrm{Da}\right)$ (Sigma-Aldrich) were evaluated as substrates for the different GtfB enzymes. Amylose V (2\%, w/v) was prepared as a stock solution in sodium hydroxide (1 M), and amylopectin and maltodextrins $(1 \%, \mathrm{w} / \mathrm{v})$ were solubilized by heating to $55{ }^{\circ} \mathrm{C}$ in Milli-Q $\mathrm{H}_{2} \mathrm{O}$. Before incubation, the amylose $\mathrm{V}$ stock solution was neutralized with $7 \mathrm{M} \mathrm{HCl}$ and diluted to a concentration of $1 \%(\mathrm{w} / \mathrm{v})$. Unless otherwise stated, the different starch-like substrates were individually incubated with $5 \mu \mathrm{g} \mathrm{mL}^{-1}$ of $\operatorname{Lr} 121 \mathrm{GtfB}$, Lr2613 GtfB, and Lf2970 GtfB at a concentration of $0.6 \%(w / v)$. All reactions were performed in $25 \mathrm{mM}$ sodium acetate buffer, $\mathrm{pH} 5.0$ with $1 \mathrm{mM} \mathrm{CaCl}$ at $37{ }^{\circ} \mathrm{C}$ for $72 \mathrm{~h}$. The reactions were stopped by incubation at $90{ }^{\circ} \mathrm{C}$ for $10 \mathrm{~min}$. The products were analyzed by highperformance size exclusion chromatography (HPSEC), proton 
nuclear magnetic resonance ( ${ }^{1} \mathrm{H}$ NMR) spectroscopy, and methylation analysis as described in the Supporting Information.

Inhibitory Effect of Acarbose on the Enzyme Activity of Lr2613 GtfB. The initial total activity of Lr2613 GtfB was assayed by the amylose-iodine staining method as described before. ${ }^{4,17}$ The reaction mixture contained $0.125 \%(\mathrm{w} / \mathrm{v})$ amylose $\mathrm{V}$ (AVEBE, Foxhol, The Netherlands), $2-100 \mu \mathrm{g} \mathrm{ml}^{-1}(0.02-1.0 \mathrm{nM})$ of enzyme in $25 \mathrm{mM}$ sodium acetate $(\mathrm{pH} 5.0)$ and $1 \mathrm{mM} \mathrm{CaCl}_{2}$. The decrease in absorbance of the $\alpha$-glucan-iodine complex resulting from transglycosylation and/or hydrolytic activity was monitored at $660 \mathrm{~nm}$ for $8 \mathrm{~min}$ at $40{ }^{\circ} \mathrm{C}$. One unit of activity was defined as the amount of enzyme converting $1 \mathrm{mg}$ of substrate per minute. The inhibitory effect of acarbose (Serva Electrophoresis GmbH, Heidelberg, Germany) on the initial total enzyme activity was measured by adding $1.0 \mathrm{mM}$ acarbose to the reaction mixture (the acarbose concentration used did not significantly affect the amylose-iodine staining assay). The initial activity of $\operatorname{Lr} 2613 \mathrm{GtfB}$ on amylose was decreased by $62 \%$ in the presence of acarbose. Incubation of the Lr2613 GtfB with acarbose revealed that this enzyme is not able to use acarbose as a substrate (data not shown).

HPSEC Analysis of Products. The molecular mass distribution of the product mixtures was determined by high-performance sizeexclusion chromatography (HPSEC) with multi-detection as described previously. ${ }^{17}$ The HPSEC system (Agilent Technologies 1260 Infinity) was equipped with a multi-angle laser light scattering detector (SLD 7000 PSS, Mainz), a viscometer (ETA-2010 PSS, Mainz), and a differential refractive index detector (G1362A 1260 RID Agilent Technologies). Separation was performed by using three PFG-SEC columns with porosities of 100,300 , and $4000 \AA$, coupled with a PFG guard column. DMSO- $\mathrm{LiBr}(0.05 \mathrm{M})$ was used as eluent

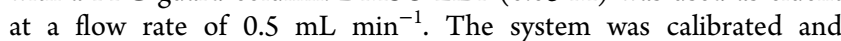
validated using a standard pullulan kit (PSS, Mainz, Germany) with $M_{\mathrm{w}}$ ranging from 342 to $708,000 \mathrm{Da}$. The specific $\mathrm{RI}$ increment value $\mathrm{d} n / \mathrm{d} c$ was also measured by PSS and was $0.072 \mathrm{~mL} \mathrm{~g}^{-1}$ (private communication with PSS). The multi-angle laser light scattering signal was used to determine the molecular mass of the high molecular mass products $\left(>1 \times 10^{5} \mathrm{Da}\right)$. The specific RI increment value, $\mathrm{d} n / \mathrm{d} c$, for these polysaccharides in this system was taken to be the same as for pullulan. The molecular mass of the low molecular mass products $\left(<1 \times 10^{5} \mathrm{Da}\right)$ was determined by a universal calibration method. WinGPC Unity software (PSS, Mainz, Germany) was used for data processing.

NMR Spectroscopy. One-dimensional ${ }^{1} \mathrm{H}$ nuclear magnetic resonance (NMR) spectra were recorded on a Varian Inova 500 spectrometer (NMR Center, University of Groningen), using $\mathrm{D}_{2} \mathrm{O}$ as solvent and at a probe temperature of $298 \mathrm{~K}$. Before the analysis, samples were exchanged twice in $\mathrm{D}_{2} \mathrm{O}$ (99.9 atom \% D, Cambridge Isotope Laboratories, Inc., Andover, MA) with intermediate lyophilization and then dissolved in $0.6 \mathrm{~mL}$ of $\mathrm{D}_{2} \mathrm{O}$. One-dimensional $500-\mathrm{MHz}{ }^{1} \mathrm{H}$ NMR spectra were recorded at a $4000 \mathrm{~Hz}$ spectral width and $16 \mathrm{k}$ complex points, using a WET1D pulse to suppress the HOD signal. All NMR spectra were processed with MestReNova 10.0.2 (Mestrelabs Research SL, Santiago de Compostella, Spain). Manual phase correction was performed and a Whittacker smoother baseline correction was applied. Chemical shifts $(\delta)$ were expressed in ppm and calibrated with the internal standard acetone $(\delta, 2.225 \mathrm{ppm})$. The percentage of different linkages was estimated by integration of the respective signal peak areas. ${ }^{6,733}$

Methylation Analysis. Methylation analysis was performed as described before. ${ }^{34}$ Briefly, the carbohydrate samples $(\sim 5 \mathrm{mg})$ were per-methylated using $\mathrm{CH}_{3} \mathrm{I}$ and solid $\mathrm{NaOH}$ in DMSO and subsequently hydrolyzed with trifluoroacetic acid $\left(2 \mathrm{~h}, 120^{\circ} \mathrm{C}\right)$. The partially methylated monosaccharides generated were reduced with $\mathrm{NaBD}_{4}(2 \mathrm{~h}$, room temperature, aqueous solution). The solution was neutralized with acetic acid and boric acid was removed by coevaporation with methanol. The resulting partially methylated alditols were per-acetylated using pyridine:acetic anhydride $(1: 1 \mathrm{v} / \mathrm{v})$ at 120 ${ }^{\circ} \mathrm{C}$ yielding mixtures of partially methylated alditol acetates (PMAAs). PMAAs were analyzed by gas-liquid chromatography (GLC) coupled to electron impact mass spectrometry (EI-MS) and GLC coupled to flame ioniziation detection (FID) as previously described. ${ }^{34}$

Crystallization and Data Collection. Crystals of Lr2613 GtfB were obtained in hanging drop vapor diffusion experiments at $293 \mathrm{~K}$, using either streakseeding or macroseeding methods. Drops for streakseeding experiments consisted of equal volumes of protein solution $\left(7 \mathrm{mg} \mathrm{ml}^{-1}\right.$ in $25 \mathrm{mM} \mathrm{HAc} / \mathrm{NaAc}, \mathrm{pH} 5.0,150 \mathrm{mM} \mathrm{NaCl}$, and $1 \mathrm{mM} \mathrm{CaCl}$ ) and reservoir solution (1.4-1.7 $\mathrm{M}\left(\mathrm{NH}_{4}\right)_{2} \mathrm{SO}_{4}$ and $0.1 \mathrm{M}$ Bis-Tris- $\mathrm{HCl}, \mathrm{pH}$ 5.5) and were equilibrated against reservoir solution. The drops were streakseeded with a cat whisker $22 \mathrm{~h}$ after set up, from previously grown crystals. Drops for macroseeding experiments consisted of equal volumes of the same protein solution and 2.7-3.1 $\mathrm{M}\left(\mathrm{NH}_{4}\right)_{2} \mathrm{SO}_{4}, 75 \mathrm{mM}$ Bis-Tris- $\mathrm{HCl}, \mathrm{pH} 5.5,25 \mathrm{mM}$ $\mathrm{HAc} / \mathrm{NaAc}$, and $150 \mathrm{mM} \mathrm{NaCl}$; they were equilibrated against reservoir solution and macroseeded immediately after set up. Crystals using either method appeared as thin plates and were often intergrown; for data collection, they were stabilized in $1.8 \mathrm{M}$ $\left(\mathrm{NH}_{4}\right)_{2} \mathrm{SO}_{4}, 20 \mathrm{mM} \mathrm{HAc} / \mathrm{NaAc}, \mathrm{pH} 5.0$, and $75 \mathrm{mM} \mathrm{NaCl}$ and cryoprotected by including 25\% (v/v) glycerol. Acarbose-complexed crystals were obtained by including 20 or $15 \mathrm{mM}$ acarbose (Serva Electrophoresis $\mathrm{GmbH}$, Heidelberg, Germany) in the stabilization and cryoprotectant solutions, respectively. Diffraction data were collected at beamlines P14 (native) and P11 (acarbose complex) of DESY (Hamburg, Germany) and indexed, integrated, and scaled using XDS; ${ }^{35}$ statistics are given in Table 1 .

Table 1. Crystallographic Data Collection and Refinement Statistics

\begin{tabular}{|c|c|c|}
\hline parameters & native & acarbose complex \\
\hline PDB entry & 7P38 & 7P39 \\
\hline \multicolumn{3}{|l|}{ data collection } \\
\hline space group & $P 2_{1} 2_{1} 2_{1}$ & $P 2_{1} 2_{1} 2_{1}$ \\
\hline $\begin{array}{l}\text { cell dimensions } a, \\
b, c(\AA)\end{array}$ & $107.4,134.5,147.9$ & $107.2,133.8,147.9$ \\
\hline resolution $(\AA)$ & $\begin{array}{l}47.24-2.70(2.77- \\
2.70)\end{array}$ & $\begin{array}{l}99.21-2.90(2.98- \\
2.90)\end{array}$ \\
\hline$R_{\text {pim }}$ & $0.130(0.606)$ & $0.141(0.551)$ \\
\hline$\langle I / \sigma\rangle$ & $6.1(1.7)$ & $3.6(1.1)$ \\
\hline $\begin{array}{l}\text { completeness } \\
(\%)^{a}\end{array}$ & $99.9(99.0)$ & $98.3(98.9)$ \\
\hline redundancy $^{a}$ & $8.2(8.3)$ & $9.1(9.0)$ \\
\hline \multicolumn{3}{|l|}{ refinement } \\
\hline resolution $(\AA)$ & $\begin{array}{l}47.24-2.70(2.77- \\
2.70)\end{array}$ & $\begin{array}{l}99.21-2.90(2.98- \\
2.90)\end{array}$ \\
\hline $\begin{array}{l}\text { unique } \\
\text { observations }{ }^{a}\end{array}$ & $56,749(4107)$ & $44,476(3254)$ \\
\hline$R / R_{\text {free }}$ & $0.260(0.299)$ & $0.293(0.315)$ \\
\hline \multicolumn{3}{|l|}{ number of atoms } \\
\hline protein & 13,001 & 13,022 \\
\hline $\mathrm{Ca}^{2+} /$ waters & $2 / 96$ & $2 / 19$ \\
\hline carbohydrate ligands & & acarbose \\
\hline other ligand molecules & $\begin{array}{l}\text { glycerol (1), sulfate } \\
\text { ion (2) }\end{array}$ & sulfate ion (1) \\
\hline \multicolumn{3}{|l|}{ B-factors } \\
\hline protein $\left(\AA^{2}\right)$ & $\begin{array}{l}39.4 \text { (molecule A), } \\
36.9 \text { (molecule B) }\end{array}$ & $\begin{array}{l}44.5 \text { (molecule A), } \\
\quad 42.3 \text { (molecule B) }\end{array}$ \\
\hline carbohydrate $\left(\AA^{2}\right)$ & & 49.1, 46.1 (acarbose) \\
\hline \multicolumn{3}{|l|}{$\begin{array}{l}\text { root-mean-square } \\
\text { deviations }\end{array}$} \\
\hline bond lengths $(\AA)$ & 0.007 & 0.007 \\
\hline bond angles $\left({ }^{\circ}\right)$ & 1.16 & 1.14 \\
\hline \multicolumn{3}{|l|}{ Ramachandran } \\
\hline favored (\%) & 93.5 & 95.3 \\
\hline allowed (\%) & 5.6 & 3.9 \\
\hline outliers (\%) & 0.9 & 0.7 \\
\hline
\end{tabular}

${ }^{a}$ In the highest resolution shell. 
Structure Determination and Refinement of Lr2613 GtfB. The crystal structure of the Lr2613 GtfB construct was determined by molecular replacement using PHASER; ${ }^{36}$ a previously obtained homology model ${ }^{6}$ based on the crystal structure of $L$. reuteri 121 $\mathrm{GtfB}(-\Delta \mathrm{N} \Delta \mathrm{V})^{16}$ was used as the search model. The asymmetric unit of the $P 2_{1} 2_{1} 2_{1}$ cell contains two molecules. Models of the native and acarbose-soaked structures were refined with Refmac ${ }^{37}$ using noncrystallographic symmetry restraints, alternated with inspection of the electron density and manual rebuilding with Coot. ${ }^{38}$ The PDB-REDO server ${ }^{39}$ was used in the final refinement stages. The final refinement statistics and model quality are listed in Table 1 . Structural figures were prepared with PyMOL (The PyMOL Molecular Graphics System, Version 2.0 Schrödinger, LLC) or UCSF ChimeraX. ${ }^{40}$ Atomic coordinates and structure factors have been deposited at the Protein Data Bank with entries 7P38 (native) and 7P39 (acarbosebound).

Homology Modeling of Lf2970 GtfB. The one-to-one Phyre2 protocol $^{41}$ was used to construct a homology model of Lf2970 GtfB, with the crystal structure of Lr2613 GtfB as the template. Residues 898-901 (in loop B) and 1114-1116 (in loop A1) were added based on the result from a normal Phyre 2 modeling protocol.

Modeling Substrate Binding. To map the substrate binding groove of all three enzymes, first the crystal structure of Lr2613 GtfB in complex with acarbose (subsites +3 to -1 ) was superimposed with that of the Lr121 GtfB-maltopentaose complex (subsites -1 to -5 ) (PDB: 5JBF). ${ }^{16}$ Guided by this superposition, a maltooctaose (G8) was then placed in subsites +3 to -5 of $\operatorname{Lr} 2613 \mathrm{GtfB}$, optimizing the substrate by adjusting glycosidic torsion angles while avoiding clashes with the protein. By superposition, this procedure was repeated for the crystal structure of Lr121 GtfB and the homology model of Lf2970 GtfB. To model a branched substrate, an $\alpha$-1,6-linked glucosyl moiety was added at the sugar unit in subsite +1 .

\section{RESULTS AND DISCUSSION}

Lr2613 GtfB, Lf2970 GtfB, and Lr121 GtfB Have Different Substrate Specificities. To study the substrate specificity of Lr2613 GtfB and Lf2970 GtfB, the product mixtures synthesized from amylose or amylopectin were analyzed regarding linkage and molecular mass distribution and compared with that of the Lr121 GtfB (Table S1). As reported before, ${ }^{18}$ Lr121 GtfB synthesizes a linear IMMP (isomalto/maltopolysaccharides). While the IMMP derived from amylose contained $80 \%$ of $\alpha-1,6$ linkages, only $14 \%$ were introduced in the amylopectin-derived products, indicating that the presence of $\alpha-1,6$ branching points in this substrate limits $\alpha-1,6$ transglycosylating activity. In contrast, Lr2613 GtfB synthesized branched reuteran-like products with similar linkage distributions for $\alpha-1,4$ and $\alpha-1,6$ (79 and 21\%, respectively) regardless of the substrate used. Finally, for Lf2970 GtfB, we analyzed the products by methylation analysis, as the anomeric signals of the $\alpha-1,3$ and $\alpha-1,4$ linked $\alpha$-D-Glcp residues partially overlap. In the product mixture synthesized from amylose, terminal, 3-substituted, 4-substituted, 3,4-disubstituted, and 4,6-disubstituted glucopyranose residues are present in molar percentages of $18,12,59,9$, and $2 \%$, respectively. In contrast, the relative amounts for the amylopectin-derived products were $22,2,69,1$, and $6 \%$, respectively.

HPSEC analysis (Figure S1) of the products synthesized from amylose or amylopectin by the three enzymes supports the linkage distribution analyses. In the case of $\operatorname{Lr} 121 \mathrm{GtfB}$, the amylose-derived products have a different molecular mass distribution than the amylopectin-derived ones, eluting as a narrow peak at $\sim 26 \mathrm{~mL}$ (corresponding to a low molecular polymer with an average $M_{\mathrm{w}}$ of $\sim 15 \times 10^{3} \mathrm{Da}$ ), or with a broader molecular mass distribution around the same $M_{\mathrm{w}}$, respectively. This HPSEC profile, together with the low amount of $\alpha-1,6$ linkages detected by ${ }^{1} \mathrm{H}$ NMR in the amylopectin-derived products (Table S1), fits with previous results reported by Bai et al. ${ }^{18}$ showing that this enzyme was only capable of modifying the linear side chains of amylopectin in wheat starches. In contrast, Lr2613 GtfB products from amylose or amylopectin showed a rather similar molecular mass distribution. The main peak at an elution volume of $\sim 29$ $\mathrm{mL}$ corresponds to a low molecular mass polymer with an average $M_{\mathrm{w}}$ of $\sim 5.6 \times 10^{3} \mathrm{Da}$, together with a small shoulder peak corresponding to maltose. Finally, for Lf2970 GtfB, the molecular mass distribution of synthesized products clearly depends on the substrate given. From amylose, Lf2970 GtfB gave a bimodal molecular mass distribution containing two main peaks corresponding to a high molecular mass polymer with an average $M_{\mathrm{w}}$ of $26 \times 10^{6} \mathrm{Da}$, and oligosaccharides. From amylopectin, however, the synthesized $\alpha$-glucans showed more complex elution patterns because of their high polydispersity indices. This suggests that Lf2970 GtfB efficiently cleaves the $\alpha-1,4$ linkages present in amylopectin, while the presence of branching points limits the synthesis of new $\alpha-1,3$ linkages leading to polymer formation. As a result, a non-uniform poly-/oligosaccharide mixture is produced from amylopectin.

Crystal Structure of Lr2613 GtfB. The native crystal structure of $\mathrm{Lr} 2613 \mathrm{GtfB}$ was determined at a resolution of 2.9 $\AA$. The asymmetric unit of the Lr2613 GtfB crystals contains two molecules (A and B), comprising residues 446-1277 and 445-1277, which can be superimposed with a root-meansquare difference (rmsd) of $0.11 \AA$ on $\mathrm{C} \alpha$ atoms. Dynamic light scattering analysis clearly indicated a monodisperse solution containing a monomeric protein (apparent molecular mass $101 \mathrm{kDa}$ ). Additional weak electron density, likely representing extra N-terminal residues of $\mathrm{Lr} 2613 \mathrm{GtfB}$, was not sufficient to include these in the model; also, the N-terminal His-tag was not visible in electron density. In the native structure, residues 805-807 of molecule B were omitted because of weak electron density. Superposition with the structure of $\operatorname{Lr} 121 \mathrm{GtfB}^{16}$ gave an rmsd of $0.42 \AA$ (for $574 \mathrm{C} \alpha$ atoms), reflecting the high sequence identity $(79.4 \%$ for the segments visible in the structures). In fact, these two GtfB structures are very similar, except for some loop regions (as will be discussed below). Lr2613 GtfB has the GH70-like domain arrangement; ${ }^{28}$ the crystal structure comprises the three core domains $\mathrm{A}, \mathrm{B}$, and $\mathrm{C}$, and the auxiliary domain IV can thus be considered a $\Delta \mathrm{N} \Delta \mathrm{V}$-structure (Figure 1). Residues preceding the first visible residue (V446), constituting domain $\mathrm{V}$ and $\mathrm{N}$-terminal variable domain $(\mathrm{N})$, likely extend away from domain IV. Like in other GH70 enzymes, the catalytic domain $\mathrm{A}$ is circularly permuted, such that the order of the four homology motifs is II-III-IV-I, differing from the GH13 I-II-III-IV homology motif order. ${ }^{24,28}$

The catalytic site of $\mathrm{Lr} 2613 \mathrm{GtfB}$ is located at the interface of domains A and B with the three catalytic residues D679 (nucleophile), E717 (acid/base), and D788 (transition-state stabilizing residue) lining a pocket that lies halfway a groove running along the domain interface; four other residues (R677, $\mathrm{H} 787, \mathrm{D} 1135$, and Q1140) that are conserved in GH70 enzymes also surround the conserved subsite -1 (Figure S2). Of these seven residues, six are also conserved in GH13 $\alpha$ amylases, while the glutamine Q1140 replaces a histidine that is present at the corresponding position in $\alpha$-amylases. Below subsite -1 is the side chain of Y1095, known to provide an 


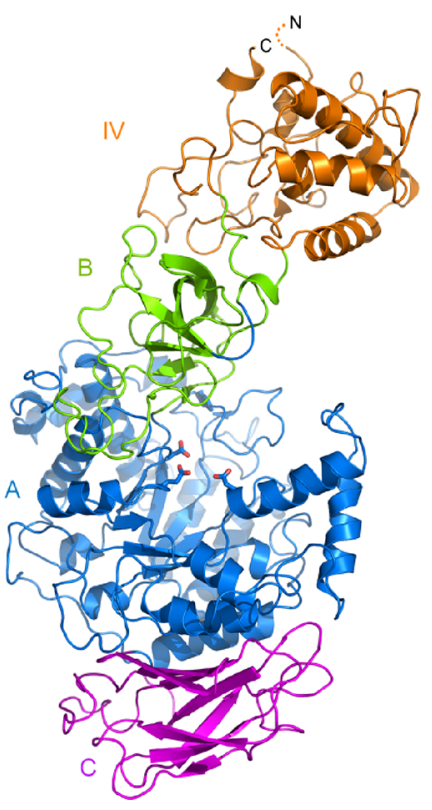

Figure 1. Crystal structure of Lr2613 GtfB- $\Delta \mathrm{N} \Delta \mathrm{V}$ with the four domains A, B, C, and IV indicated. The active site is at the interface of domains $\mathrm{A}$ and $\mathrm{B}$, with the three catalytic residues (D679, E717, and D788) shown in stick representation. The figure is prepared with PyMOL. aromatic stacking interaction with sugar moieties in this subsite. ${ }^{24}$ Close to the active site is a conserved $\mathrm{Ca}^{2+}$ binding site also found in other GH70 enzyme structures.

Most notably, the Lr2613 GtfB structure shows an open and fully accessible binding groove. In agreement with earlier modeling, ${ }^{6}$ the short loops A1 (residues 802-811) and B (residues 590-593) do not form a tunnel; details and comparison with other GtfB structures are discussed below (section "GtfB Enzymes Display Varying A1 and B Loop Lengths and Binding Groove Architectures").

The Lr2613 GtfB-Acarbose Complex Reveals Acceptor Binding Subsites. Acarbose is a known inhibitor of GH13 and GH70 enzymes and the inhibitory effect on Lr2613 GtfB activity was confirmed (Supporting Information). Soaking the crystals with acarbose resulted in binding of the pseudotetrasaccharide in subsites -1 to +3 (Figure 2). The valeinamine moiety at the non-reducing end of the inhibitor is bound in subsite -1 where, in case of a natural substrate (e.g., a maltooligosaccharide fragment), the covalent enzyme-glucosyl intermediate is formed in the first half-reaction. The valeinamine moiety has a distorted ${ }^{2} \mathrm{H}_{3}$ conformation, mimicking the transition state, ${ }^{42}$ with the non-cleavable $\mathrm{N}$-glycosidic linkage oriented toward the catalytic acid/base residue E717. A total of seven hydrogen bonds to the valeinamine moiety and its $\mathrm{N}$ glycosidic linkage are provided by two of the three catalytic residues (acid/base E717 and D788) as well as the strictly conserved H787. The orientation of this sugar moiety is further stabilized by an aromatic stacking interaction with a
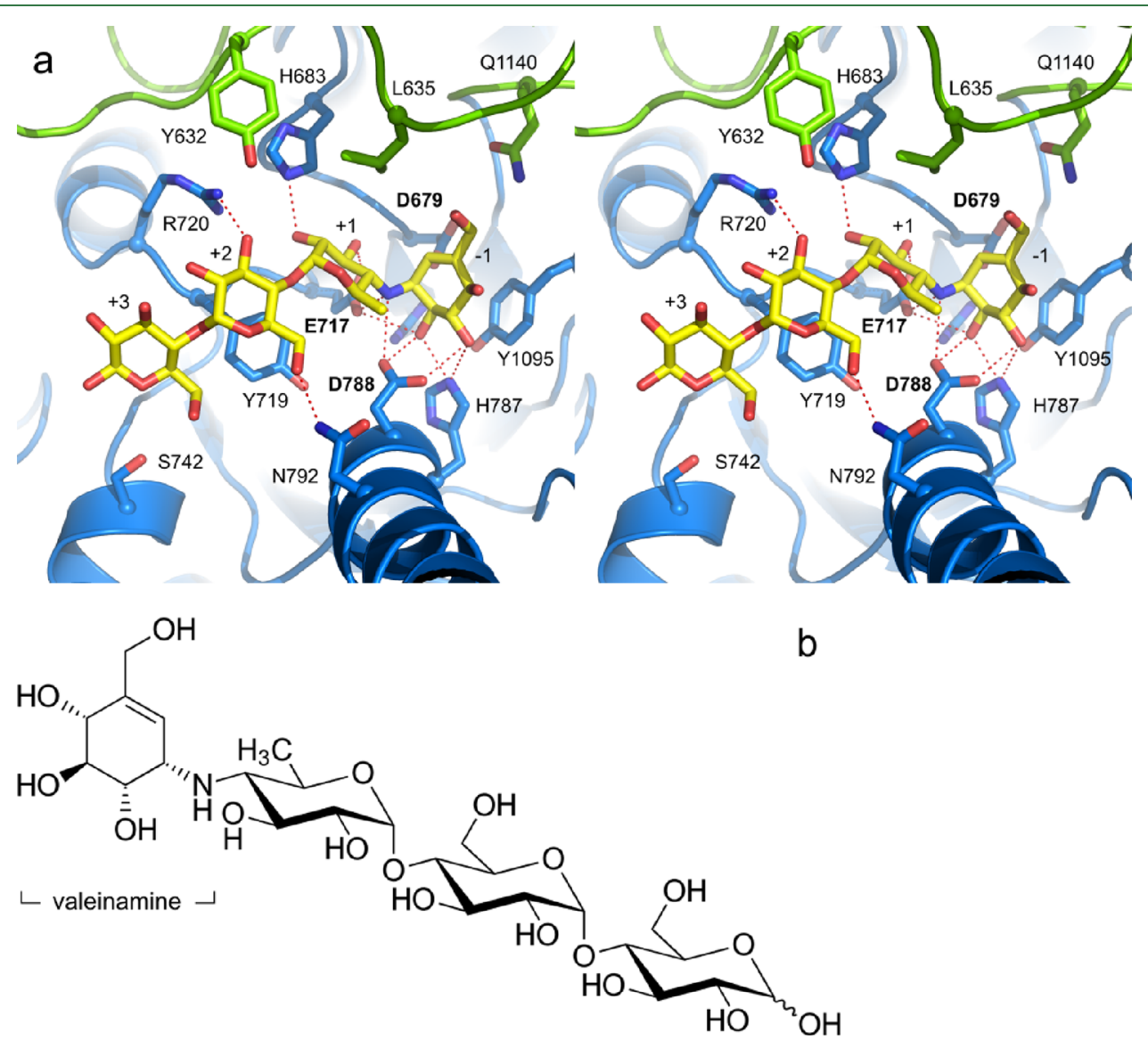

Figure 2. (a) Stereo view of the Lr2613 GtfB-acarbose complex, with the pseudotetrasaccharide inhibitor occupying donor subsite -1 and acceptor subsites +1 to +3 . Residues close to acarbose are shown with side chains (catalytic residues D679, E717, and D788 are indicated with bold labels); hydrogen bond interactions are shown as dotted lines. The figure is prepared with PyMOL. (b) Chemical structure of acarbose. 


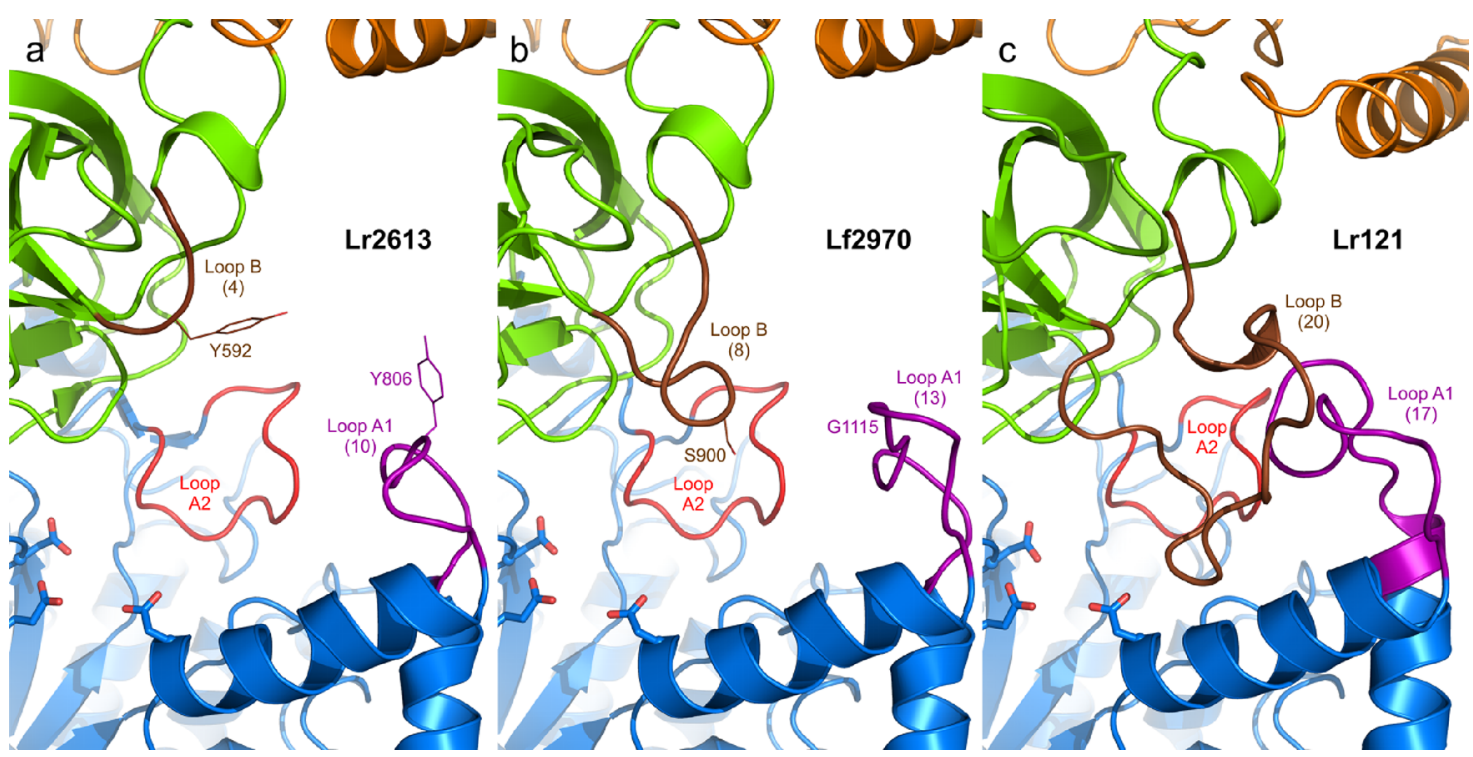

Figure 3. (a-c) Comparison of loops A1 (purple), A2 (red), and B (brown) in the crystal structure of Lr2613 GtfB (this study), the homology model of Lf2970 GtfB (this study), and the crystal structure of Lr121 GtfB. ${ }^{16}$ The three loops are labeled with their lengths; in the first two GtfBs, amino acid residues at the tip of loops $\mathrm{A} 1$ and $\mathrm{B}$ are indicated. The catalytic residues (D679, E717, and D788) are shown in stick representation (lower left). In Lr2613 GtfB and Lf2970 GtfB, loops A1 and B are relatively short, leading to a (half-)open binding groove architecture; in contrast, in Lr121 GtfB the corresponding segments are long and cover donor subsites. The figure is prepared with PyMOL.

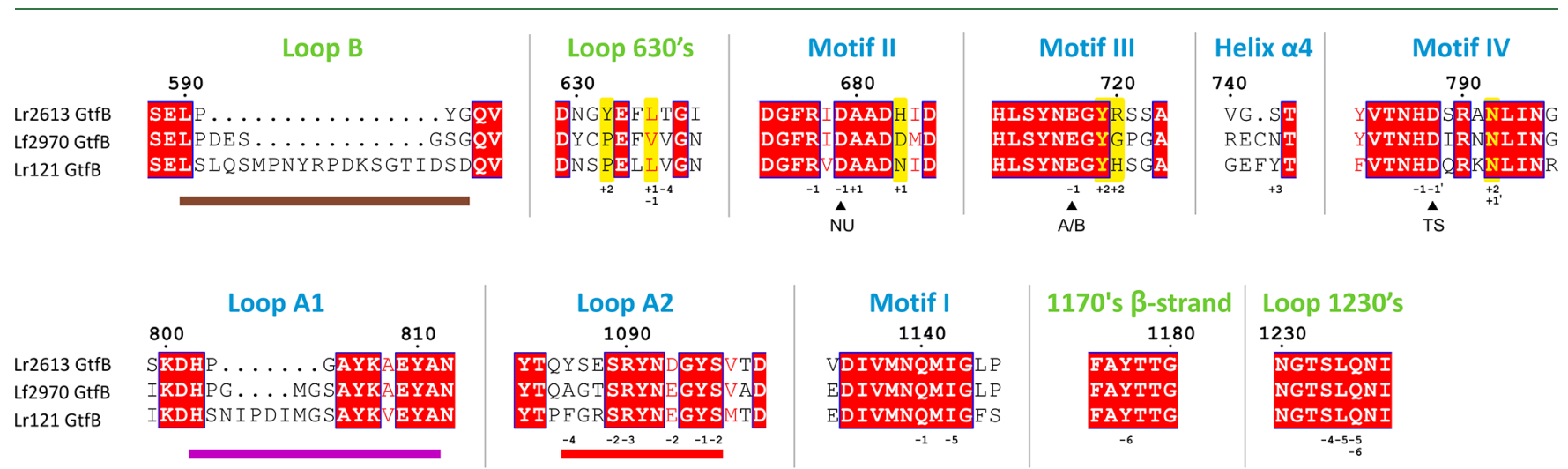

Figure 4. Sequence alignment of regions in Lr2613 GtfB, Lf2970 GtfB, and Lr121 GtfB in domains A (blue header) and B (green header) in which residues closer than $4 \AA$ to a modeled substrate are shown below the alignment with the corresponding subsite. The six acceptor subsite residues discussed in the text are highlighted in yellow. The three catalytic residues (D679, E717, and D788) are indicated with a black triangle (NU = nucleophile, $\mathrm{A} / \mathrm{B}=$ general acid/base, TS = transition-state stabilizing residue). Color coding of the loops $\mathrm{B}, \mathrm{A} 1$, and $\mathrm{A} 2$ corresponds to the scheme used in Figure 3. The figure is prepared using the ESPript 3.0 server (https://espript.ibcp.fr). ${ }^{45}$

conserved tyrosine residue (Y1095). The remaining three sugar moieties occupy subsites +1 to +3 and, for the first time, map these three acceptor substrate subsites in a GH70 GtfBtype enzyme. Surrounding these subsites, there are six residues (apart from the catalytic residues) closer than $4 \AA$ from the bound inhibitor, shaping the binding site and providing a hydrogen bond or aromatic stacking interactions at subsites +1 and +2 ; they will be discussed below. No interactions were observed at subsite +3 , although we cannot exclude that watermediated hydrogen bonds are present, which are unresolved at 2.9 Å resolution.

Improved Homology Model of Lf2970 GtfB. The previously reported homology model of $\mathrm{Lf} 2970 \mathrm{GtfB}^{7}$ was based on the crystal structure of glucansucrase Gtf180- $\Delta \mathrm{N}$ from $L$. reuteri 180 (sequence identity: $38.6 \%$ for segments visible in the structure). Because of the much higher sequence identity with Lr2613 GtfB (93.7\%), the homology model of Lf2970 GtfB presented here is more accurate. The new model comprises residues $752-1589$ of Lf2970 GtfB; with a virtually identical chain trace, superposition with molecule $A$ of the Lr2613 GtfB crystal structure resulted in an rmsd of only 0.13 $\AA$ (for $750 \mathrm{C} \alpha$ atoms). The most notable differences occur in loops A1 and B; how the Lf 2970 GtfB binding groove compares to that of the two other GtfB enzymes will be reviewed below.

GtfB Enzymes Display Varying A1 and B Loop Lengths and Binding Groove Architectures. Comparing the two crystal structures (Lr2613 GtfB and Lr121 GtfB) and an improved homology model (Lf2970 GtfB) highlights the differences in the architecture of the binding groove and the importance of loops A1 and B for accessibility, as is shown in Figure 3. Together with two other surface loops (around D601 and N625 (Lr2613 GtfB numbering), too far from the active site to be involved in substrate binding), loops A1 and B map to gaps in the sequence alignment. In Lr2613 GtfB, these loops are much shorter than in Lr121 GtfB, namely, by 7 and 16 
residues, respectively (Figures $3 a$ and 4 ). As a result, and in stark contrast to Lr121 GtfB (Figure 3c), loop B hardly protrudes from the surface; in fact, only a non-conserved residue (Y592) does so. Also loop A1 (residues H802-Y810) protrudes considerably less from the surface than in $\operatorname{Lr} 121$ GtfB.

As a result, and as was previously predicted from sequence alignment and homology modeling, ${ }^{6}$ the crystal structure of Lr2613 GtfB now clearly shows that the shorter loops A1 and $\mathrm{B}$ do not form a tunnel (the hydroxyl groups of residues Y806 and Y592 at their tips are about $9 \AA$ apart); this creates a much more open architecture. It has to be noted that the electron density for residues K800-A811 is weak and/or ambiguous in both molecules, suggesting possible flexibility of loop A1. Nevertheless, the active site groove of Lr2613 GtfB is fully accessible, reminiscent of the situation in GH13 starchdegrading $\alpha$-amylases. ${ }^{43}$ In Lf2970 GtfB, loops A1 and B are somewhat longer than in Lr2613 GtfB (by three and four residues, respectively) and loop $B$ protrudes more from the surface (Figure $3 \mathrm{~b}$ ). Still, the residues at the tips ( 900 and G1115) are at such a distance that Lf2970 GtfB displays a (half-)open architecture.

In order to extend our analysis of loop length beyond these three enzymes to the entire GtfB subfamily, we performed a BLAST search with the core domains (A, B, and C) of Lr121 GtfB. This yielded 287 putative GtfB enzyme sequences (Table S2), including the three enzymes described in our study; the vast majority of these (280) are from Lactobacillaceae, while the rest are from Weissella (5), Leuconostoc (2), or Streptococcus (1) species. The sequence identity of the hits ranged from 99.9 to $44.2 \%$. Given the importance of loops A1 and $\mathrm{B}$ in determining the accessibility of the substrate binding groove (especially near donor subsites) and knowing that in characterized GtfB enzymes their lengths differ, we focused on the regions corresponding to these loops. Indeed, sequence analysis and alignment of loops A1 and B in 287 putative GtfB enzymes revealed a large variation in the length of these elements with a rather distinct distribution (Figure 5); within groups of enzymes having similar loop lengths, the loop sequences are highly conserved (not shown).

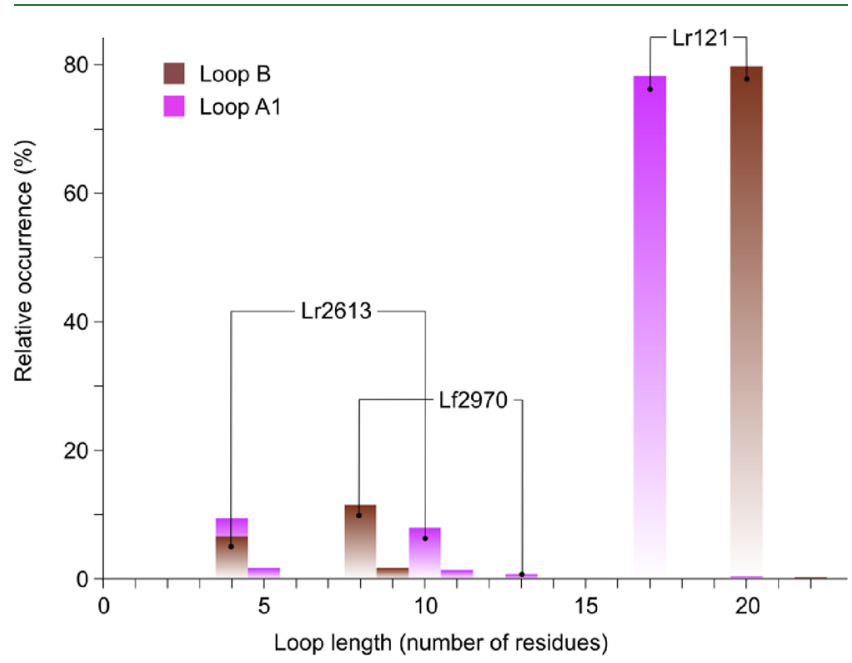

Figure 5. Variation in the length of loops A1 (purple) and B (brown) in 287 putative GH70 GtfB enzymes. The groups in which Lr2613-, Lf2970-, and Lr121-GtfB occur are indicated (e.g., Lr2613 GtfB has a four-residue-long loop B and a 10-residue-long loop A1).
The most common length for loop A1 is 17 residues $(78.4 \%$ of cases), while most of the other sequences feature a much shorter loop A1. The length of loop B varies between 4 and 20 residues, again with the majority ( $80.1 \%$ of cases) having the long version. The distribution of total loop length $(\mathrm{A} 1+\mathrm{B})$ is similar, with two distinct groups: $78.4 \%$ (225 sequences) of the enzymes feature long loops (37 or 40 residues combined), while most of the remaining ones have shorter loops $(17.8 \%$ have a combined length of 12-14 residues). The three enzymes that we compared structurally represent GtfBs with (almost) the shortest (Lr2613 GtfB: 14 residues), intermediate (Lf2970 GtfB: 21 residues), and (almost) the longest combined loop lengths (Lr121 GtfB: 37 residues). The small subset with intermediate loop lengths includes $S$. thermophilus GtfB (combined loop length of 21 residues). A phylogenetic tree (Figure S3) constructed from the 287 putative GtfB sequences, annotated with the total loop length $(A 1+B)$, revealed that those with short loops cluster together in two opposite segments, one mostly containing Lactiplantibacillus plantarum or Fructilactobacillus sanfranciscensis species, and the other mainly containing Limosilactobacillus reuteri and other species (e.g., Limosilactobacillus fermentum).

Modeling Substrate Binding. The first step of the reaction catalyzed by GTs is donor substrate binding; we combined structural data from two GtfB enzymes to gain more insights into the molecular details. Superposition of the Lr2613 GtfB-acarbose complex with the Lr121 GtfB-maltopentaose complex (PDB: $5 \mathrm{JBF}^{16}$ ) showed that the (pseudo)sugar units in subsite -1 have a similar orientation and almost all hydrogen bond interactions are conserved. This allowed us to construct a model of maltooctaose (G8) occupying subsites -5 to +3 in Lr2613 GtfB (Figure 6b); only minor adjustments of glycosidic torsion angles were required to fit the oligosaccharide substrate in the binding groove. Similarly, we modeled maltooctaose in the homology model of Lf2970 GtfB (Figure $6 \mathrm{c}$ ) and in the crystal structure of Lr121 GtfB (Figure 6d), visualizing how linear $\alpha$-1,4-linked maltooligosaccharide segments of starch substrates can be bound. Comparing our models with the crystal structure of the $\alpha$-amylase fromBacillus halmapalus complexed with a pseudo-nonasaccharide (PDB: 1W9X ${ }^{43}$ (Figure 6a) showed that the global binding mode of a maltooligosaccharide substrate in $\mathrm{GH} 70 \mathrm{GtfBs}$ is similar to that observed in this $\alpha$-amylase.

After modeling a bound linear donor substrate in GtfB-type proteins, we investigated the possibility to model $\alpha-1,4 / 6$ branched ones. As described above, the open architecture of the binding groove of Lr2613 GtfB differs considerably from that of the tunneled one observed in Lr121 GtfB. The (linear) G8 models show that the positions of the O6 atoms of the glucosyl moieties in several subsites are such that a branched structure would fit without sterical hindrance in Lr2613 GtfB but not in Lr121 GtfB. Therefore, analogous to the observed binding mode of a branched oligosaccharide in the GH13 pullulanase SusG (PDB: $6 \mathrm{BS6}^{44}$ ), we modeled a branch glucosyl unit linked to the $\mathrm{O} 6$ at subsite +1 (Figure S4), showing that the branch unit may interact with residue N792 in homology motif IV, the fourth residue after the transitionstate stabilizing aspartate (D788), and almost fully conserved in the GtfB subfamily (Figure 7). In SusG, such an interaction is indeed experimentally observed with the corresponding residue K541. The orientation of the branch unit is such that the branch can be extended via $\alpha-1,4$ linkages without sterical hindrance. In Lf $2970 \mathrm{GtfB}$, the situation is very similar since 

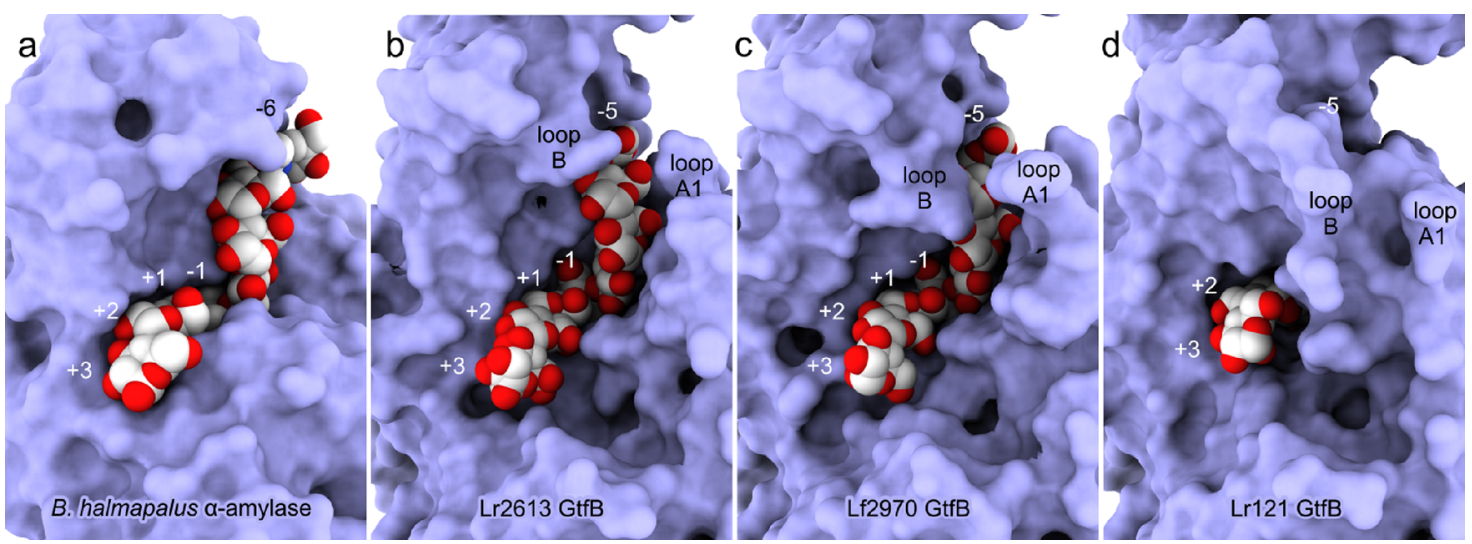

Figure 6. (b-d) Modeled maltooctaose shown as spheres in subsites +3 to -5 of Lr2613 GtfB, Lf2970 GtfB, and Lr121 GtfB, compared to (a) the structure of $B$. halmapalus $\alpha$-amylase with a bound nonasaccharide. ${ }^{43}$ With shorter loops A1 and B, Lr2613 GtfB and Lf2970 GtfB feature a more open architecture like in the $\alpha$-amylase, compared to the tunneled one in $\operatorname{Lr} 121 \mathrm{GtfB}$. The figure is prepared with $\mathrm{UCSF}^{\mathrm{ChimeraX}}{ }^{40}$

GH13 $\alpha$-amylases

B. licheniformis a-amylase
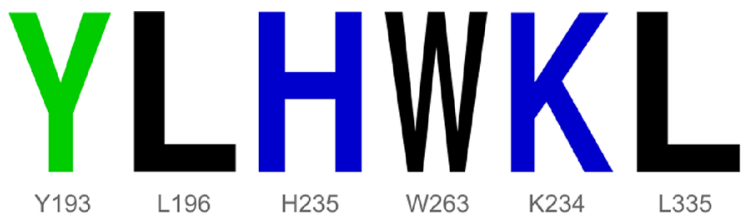

\section{Short/intermediate loops A1+B}

(57 seq.)

\section{Lr2613 GtfB (short)} Lf2970 GtfB (interm.)
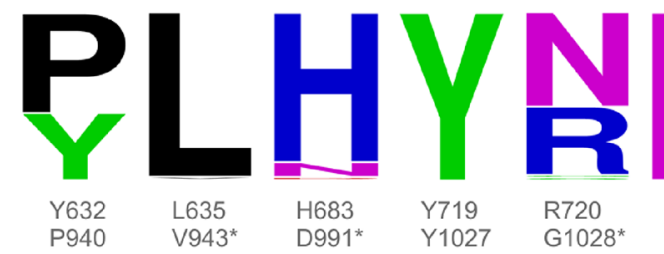

\section{GH70 GtfB \\ Long loops A1+B}

(230 seq.)

Lr121 GtfB (long)
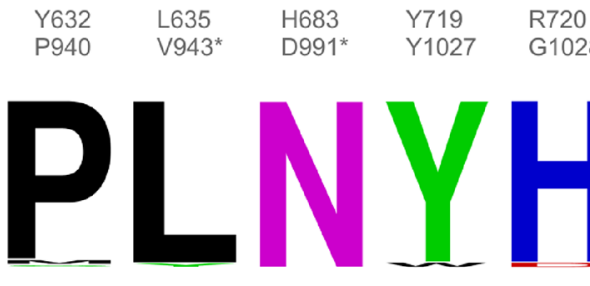

G1028*

P968

L971

N1019

Y1055

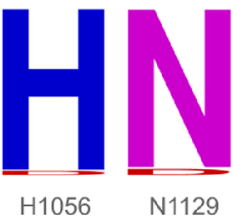

Figure 7. Sequence conservation of six residues near acceptor subsites +1 and +2 in 287 putative GH70 GtfB enzymes and GH13 $\alpha$-amylases. The $\mathrm{GtfB}$ enzymes are divided in 57 sequences with short or intermediate combined loop lengths (12-21 residues) and 230 sequences with long lengths (31-40 residues). In $\alpha$-amylases, the six corresponding residues are fully conserved. Below the logos, the residues present in $B$. licheniformis $\alpha$-amylase, Lr2613 GtfB, Lf2970 GtfB, and Lr121 GtfB are shown; the three residues in Lf2970 GtfB that are unique are indicated with an asterisk. The figure is based on use of the WebLogo server. ${ }^{29}$

the only main differences (somewhat longer loops A1 and B) are far from subsite +1 . In contrast, as is clear from Figure $S 4$, in Lr121 GtfB, residues in the region S918-D922 of loop B would clash with branch sugar units in subsite $+1^{\prime}$, hindering the binding of branched starch substrates.

Acceptor Subsite Residues Correlate with Loop Lengths. In addition to the architecture of the binding groove, residues surrounding acceptor subsites are of importance for the specificity of the transglycosylation reaction, since they determine the orientation of bound acceptor molecules after formation of the covalent glycosylenyme intermediate. The Lr2613 GtfB-acarbose complex as well as the modeled G8 substrate complex in all three enzymes show that at subsites +1 to +3 , there are six residues (apart from the catalytic residues) that are at less than $4 \AA$ distance from the sugar moieties (Figures 2 and 4). Four of these residues are from homology motifs II, III, and IV: residues H683, R720, and N792 provide hydrogen bond interactions with hydroxyl groups of (pseudo)sugar moieties in subsites +1 , $+1^{\prime}$ (in the case of a branched substrate), and +2 , while Y719 in motif III provides an aromatic stacking interaction at subsite +2. Finally, residues from the 630's loop in domain B, especially Y632 and L635, do not directly interact with the inhibitor but they help shape the binding site. Analysis of the conservation of these six residues in 287 putative GtfB sequences revealed that those corresponding to positions 635, 719 , and 792 (Lr2613 GtfB numbering) are almost fully conserved, while the others show strong preference for a certain residue type, remarkably correlating with the loop length (Figure 7). Sequences with long loops A1 and B almost invariantly have $\mathrm{P}, \mathrm{L}, \mathrm{N}, \mathrm{Y}, \mathrm{H}$, and $\mathrm{N}$ (e.g., Lr121 GtfB is representative of this group), while in sequences with short or intermediate loop lengths, the first, third, and fifth residues are often $\mathrm{Y}, \mathrm{H}$, and $\mathrm{R} / \mathrm{N}$, respectively. The residues at these latter positions $(632,683$, and 720 in $\mathrm{Lr} 2613 \mathrm{GtfB})$ cluster together near subsites +1 and +2 , and one may speculate that a different set of residues (with different hydrogen bond capabilities) is needed to process starch(-like) substrates in a more open 
binding groove. In fact, a superposition with bacterial $\alpha$ amylase structures (not shown) revealed that the corresponding residues are invariably $\mathrm{Y}, \mathrm{H}$, and $\mathrm{K}$. Together, these observations suggest that the GH70 GtfB subgroup with short loops are evolutionary closer to GH13 $\alpha$-amylases, while the large subgroup with long loops have evolved further away. The GtfB subgroup with open architecture (short loops A1 and B) therefore provides an opportunity to study how they gained transglycosylation capabilities (most $\alpha$-amylases are hydrolytic).

The six aforementioned residues likely also contribute to transglycosylation linkage specificity. Most of the GtfB enzymes characterized so far display $\alpha-4,6$ specificity, ${ }^{3,6,9}$ but Lf2970 GtfB is the only known GT with $\alpha-4,3$ transglycosylation specificity. ${ }^{7}$ Notably, within our set of 287 putative $\mathrm{GtfB}$ sequences, Lf2970 GtfB has unique substitutions at the second, third, and fifth positions (V943, D991, and G1028) (Figure 7); one may speculate that they play a key role in determining the orientation of acceptor substrates and thus the type of glycosidic linkage in the product. Preliminary modeling of acceptor substrates complexes in Lr2613 GtfB and Lf2970 GtfB so far did not give conclusive insight into this regard nor did single mutations targeting all six positions in Lr2613 GtfB affect the transglycosylation linkage specificity (personal communication). Possibly, simultaneous mutation of several residues is needed to achieve such changes.

In addition to transglycosylation linkage specificity, the acceptor subsite residues may also play a role in determining the branching characteristics of the products. An interesting comparison in this regard is the pair of GtfB-type enzymes $\mathrm{GtfY}$ and GtfX from Ligilactobacillus aviarius subsp. aviarius DSM 20655 of which $\Delta \mathrm{N} \Delta \mathrm{C}$-constructs were characterized by Meng et al. ${ }^{9}$ While GtfY was shown to synthesize linear (IMMP) products from amylose $\mathrm{V}$, the GtfX product is a branched reuteran. Notably, within our set of 287 putative GtfBs, GtfY belongs to the majority with P, L, N, Y, H, and N in the six positions near acceptor subsites, while GtfX belongs to a small set of seven sequences (all from $L$. aviarius species) having $\mathrm{M}, \mathrm{Y}, \mathrm{N}, \mathrm{W}, \mathrm{D}$, and $\mathrm{D}$ at these positions (Figure 7). This would suggest a role of these residues in facilitating branching in GtfX, even more so because both enzymes feature long loops A1 and B (17 and 20 residues, respectively (Table S2)). It has to be noted that one would expect the long loops to sterically hinder branch formation at the subsite +1 sugar moiety just like in Lr121 GtfB. In any case, at least for this small set of $L$. aviarius enzymes, loop architecture does not entirely define product specificity.

Implications for GtfB Reaction Specificity. Together, the observed differences between the Lr2613-, Lf2970-, and Lr121 GtfB structures further support the hypothesis stated by Bai et al. ${ }^{16}$ that the evolution of GH13 $\alpha$-amylases toward GH70 $\alpha$-glucanotransferases involved changes in loop architecture that conferred changes in starch substrate preference (and product specificity). For these three enzymes, their binding groove architectures are in agreement with experimental biochemical observations; while Lr121 GtfB has a profound preference for linear starch substrates, Lr2613 GtfB and Lf2970 process both linear and branched ones, albeit that with the latter enzyme, the $\alpha$-glucan product size and linkage distribution varies with the amount of branching in the substrate (Table S1). Given the high sequence similarity of loops $\mathrm{A} 1$ and $\mathrm{B}$ (within groups of enzymes having similar loop lengths) and having analyzed the $3 \mathrm{D}$ structure of three representative GtfB $\alpha$-glucanotransferases, we hypothesize that the majority (four out of five) of GtfB enzymes feature a tunnel-like structure like $\operatorname{Lr} 121 \mathrm{GtfB}$ and thus are limited to processing mostly linear starch-like structures. In contrast, most other GtfB enzymes feature short loops A1 and B and can be expected to have broader substrate specificity, allowing also more branched starches to be processed. This needs to be validated in the future by biochemical characterization of more $\mathrm{GtfB}$ enzymes. It is remarkable that intermediate loop lengths are hardly observed, and that GtfB enzymes with long loops A1 and $B$ moreover feature a different set of acceptor subsite residues. This suggests that most $\mathrm{GH} 70 \mathrm{GtfBs}$ have evolved away from starch-degrading GH13 $\alpha$-amylases, which have an open binding groove, and perhaps have reached optimal loop lengths ( $\sim 37$ residues). It is yet unclear what was the evolutionary drive for such changes, but it has been suggested that a tunneled binding groove enhances the processivity of $\alpha$ glucan synthesis ${ }^{16}$ by keeping intermediate reaction products bound to the enzyme. Indeed, when comparing the product sizes of Lr2613 GtfB (short loops) and Lr121 GtfB (long loops), the latter synthesizes higher molecular mass products from the same substrate (Figure S1). In any case, the quest for GH70 $\alpha$-glucanotransferases with broad starch substrate specificity should focus on enzymes with shorter A1 and B loops. The recent findings that Streptococcus thermophilus GtfB, with loop lengths similar to Lf $2970 \mathrm{GtfB}$, can be used in potato starch and wheat starch conversion ${ }^{11,12}$ supports this hypothesis. We are currently investigating the suitability of Lf2970- and Lr2613 GtfB for the modification of different food starches, aiming to extend the GtfB toolkit for starch modification.

\section{ASSOCIATED CONTENT}

\section{Supporting Information}

The Supporting Information is available free of charge at https://pubs.acs.org/doi/10.1021/acs.jafc.1c05657.

(Table S1) Evaluation of different starch-like substrates (amylose $\mathrm{V}$ and amylopectin) as donor substrates for different GtfB enzymes, (Figure S1) distribution of the molecular mass of products synthesized by Lr121 GtfB, Lr2613 GtfB, and Lf2970 GtfB from amylose and amylopectin. (Figure S2) stereo view of the Lr2613 GtfB active site at the interface of domains $\mathrm{A}$ and $\mathrm{B}$, (Figure S3) phylogenetic tree of 287 putative GtfB sequences, and (Figure S4) modeling of a branched maltooligosaccharide in the substrate binding sites of $\mathrm{Lr} 2613 \mathrm{GtfB}$, Lf2970 GtfB, and Lr121 GtfB. (Table S2) List of 287 putative GtfB enzymes (PDF)

\section{AUTHOR INFORMATION}

\section{Corresponding Author}

Tjaard Pijning - Biomolecular X-ray Crystallography, Groningen Biomolecular Sciences and Biotechnology Institute (GBB), University of Groningen, Groningen 9747 AG, The Netherlands; 이이.org/0000-0003-4107-3663; Email: t.pijning@rug.nl

\section{Authors}

Joana Gangoiti - Microbial Physiology, Groningen Biomolecular Sciences and Biotechnology Institute (GBB), University of Groningen, Groningen 9747 AG, The Netherlands 
Evelien M. te Poele - Microbial Physiology, Groningen Biomolecular Sciences and Biotechnology Institute (GBB), University of Groningen, Groningen 9747 AG, The Netherlands; CarbExplore Research B.V., Groningen 9747 $A A$, The Netherlands

Tim Börner - Nestlé Research, Société des Produits Nestlé SA, 1000 Lausanne, Switzerland; 이이.org/0000-00031120-225X

Lubbert Dijkhuizen - Microbial Physiology, Groningen Biomolecular Sciences and Biotechnology Institute (GBB), University of Groningen, Groningen 9747 AG, The Netherlands; CarbExplore Research B.V., Groningen 9747 $A A$, The Netherlands

Complete contact information is available at: https://pubs.acs.org/10.1021/acs.jafc.1c05657

\section{Funding}

This work was financially supported by Nestec Ltd. and by the University of Groningen.

\section{Notes}

The authors declare no competing financial interest.

\section{ACKNOWLEDGMENTS}

Parts of this research were carried out at PETRA III at DESY, a member of the Helmholtz Association (HGF). We would like to thank E. Reddem, A. Burkhardt, and G. Bourenkov for assistance in using the P11 and P14 beamlines.

\section{REFERENCES}

(1) Lombard, V.; Golaconda Ramulu, H.; Drula, E.; Coutinho, P. M.; Henrissat, B. The carbohydrate-active enzymes database (CAZy) in 2013. Nucleic Acids Res. 2014, 42, D490-D495.

(2) Kralj, S.; Grijpstra, P.; van Leeuwen, S. S.; Leemhuis, H.; Dobruchowska, J. M.; van der Kaaij, R. M.; Malik, A.; Oetari, A.; Kamerling, J. P.; Dijkhuizen, L. 4,6- $\alpha$-glucanotransferase, a novel enzyme that structurally and functionally provides an evolutionary link between Glycoside Hydrolase enzyme Families 13 and 70. Appl. Environ. Microbiol. 2011, 77, 8154-8163.

(3) Leemhuis, H.; Dijkman, W. P.; Dobruchowska, J. M.; Pijning, T.; Grijpstra, P.; Kralj, S.; Kamerling, J. P.; Dijkhuizen, L. 4,6- $\alpha$ Glucanotransferase activity occurs more widespread in Lactobacillus strains and constitutes a separate GH70 subfamily. Appl. Microbiol. Biotechnol. 2013, 97, 181-193.

(4) Gangoiti, J.; Pijning, T.; Dijkhuizen, L. The Exiguobacterium sibiricum 255-15 GtfC enzyme represents a novel Glycoside Hydrolase 70 subfamily of 4,6- $\alpha$-glucanotransferase enzymes. Appl. Environ. Microbiol. 2016, 82, 756-766.

(5) Gangoiti, J.; van Leeuwen, S. S.; Vafiadi, C.; Dijkhuizen, L. The Gram-negative bacterium Azotobacter chroococcum NCIMB 8003 employs a new glycoside hydrolase family $704,6-\alpha$-glucanotransferase enzyme (GtfD) to synthesize a reuteran like polymer from maltodextrins and starch. Biochim. Biophys. Acta 2016, 1860, 12241236.

(6) Gangoiti, J.; van Leeuwen, S. S.; Meng, X.; Duboux, S.; Vafiadi, C.; Pijning, T.; Dijkhuizen, L. Mining novel starch-converting Glycoside Hydrolase 70 enzymes from the Nestle Culture Collection genome database: The Lactobacillus reuteri NCC 2613 GtfB. Sci. Rep. 2017, 7, 9947.

(7) Gangoiti, J.; van Leeuwen, S. S.; Gerwig, G. J.; Duboux, S.; Vafiadi, C.; Pijning, T.; Dijkhuizen, L. 4,3- $\alpha$-Glucanotransferase, a novel reaction specificity in glycoside hydrolase family 70 and clan GH-H. Sci. Rep. 2017, 7, 39761.

(8) Gangoiti, J.; Lamothe, L.; van Leeuwen, S. S.; Vafiadi, C.; Dijkhuizen, L. Characterization of the Paenibacillus beijingensis DSM $24997 \mathrm{GtfD}$ and its glucan polymer products representing a new glycoside hydrolase 70 subfamily of 4,6- $\alpha$-glucanotransferase enzymes. PLoS One 2017, 12, No. e0172622.

(9) Meng, X.; Gangoiti, J.; de Kok, N.; van Leeuwen, S. S.; Pijning, T.; Dijkhuizen, L. Biochemical characterization of two GH70 family 4,6- $\alpha$-glucanotransferases with distinct product specificity from Lactobacillus aviarius subsp. aviarius DSM 20655. Food Chem. 2018, 253, 236-246.

(10) İspirli, H.; Şimşek, Ö.; Skory, C.; Sağdıç, O.; Dertli, E. Characterization of a 4,6- $\alpha$-glucanotransferase from Lactobacillus reuteri E81 and production of malto-oligosaccharides with immunemodulatory roles. Int. J. Biol. Macromol. 2019, 124, 1213-1219.

(11) Li, X.; Fei, T.; Wang, Y.; Zhao, Y.; Pan, Y.; Li, D. Wheat starch with low retrogradation properties produced by modification of the GtfB enzyme 4,6- $\alpha$-glucanotransferase from Streptococcus thermophilus. J. Agric. Food Chem. 2018, 66, 3891-3898.

(12) Li, D.; Fu, X.; Mu, S.; Fei, T.; Zhao, Y.; Fu, J.; Lee, B. H.; Ma, Y.; Zhao, J.; Hou, J.; Li, X.; Li, Z. Potato starch modified by Streptococcus thermophilus GtfB enzyme has low viscoelastic and slowly digestible properties. Int. J. Biol. Macromol. 2021, 183, 12481256.

(13) Meng, X.; Gangoiti, J.; Bai, Y.; Pijning, T.; Van Leeuwen, S. S.; Dijkhuizen, L. Structure-function relationships of family GH70 glucansucrase and 4,6- $\alpha$-glucanotransferase enzymes, and their evolutionary relationships with family GH13 enzymes. Cell. Mol. Life Sci. 2016, 73, 2681-2706.

(14) Janeček, S.; Svensson, B.; MacGregor, E. A. $\alpha$-Amylase: an enzyme specificity found in various families of glycoside hydrolases. Cell. Mol. Life Sci. 2014, 71, 1149-1170.

(15) Miao, M.; Jiang, B.; Jin, Z.; BeMiller, J. N. Microbial starchconverting enzymes: recent insights and perspectives. Compr. Rev. Food Sci. Food Saf. 2015, 17, 1238-1260.

(16) Bai, Y.; Gangoiti, J.; Dijkstra, B. W.; Dijkhuizen, L.; Pijning, T. Crystal structure of 4,6- $\alpha$-glucanotransferase supports diet-driven evolution of GH70 enzymes from $\alpha$-amylases in oral bacteria. Structure 2017, 25, 231-242.

(17) Bai, Y.; van der Kaaij, R. M.; Leemhuis, H.; Pijning, T.; van Leeuwen, S. S.; Jin, Z.; Dijkhuizen, L. Biochemical Characterization of the Lactobacillus reuteri Glycoside Hydrolase Family 70 GTFB type of 4,6- $\alpha$-glucanotransferase enzymes that synthesize soluble dietary starch fibers. Appl. Environ. Microbiol. 2015, 81, 7223-7232.

(18) Bai, Y.; Boger, M.; van der Kaaij, R. M.; Woortman, A. J. J.; Pijning, T.; van Leeuwen, S. S.; Lammerts van Bueren, A.; Dijkhuizen, L. Lactobacillus reuteri strains convert starch and maltodextrins into homoexopolysaccharides using an extracellular and cell-associated 4,6$\alpha$-glucanotransferase. J. Agric. Food Chem. 2016, 64, 2941-2952.

(19) van der Zaal, P. H.; Schols, H. A.; Bitter, J. H.; Buwalda, P. L. Isomalto/malto-polysaccharide structure in relation to the structural properties of starch substrates. Carbohydr. Polym. 2018, 185, 179186

(20) Leemhuis, H.; Dobruchowska, J. M.; Ebbelaar, M.; Faber, F.; Buwalda, P. L.; van der Maarel, M. J. E. C.; Kamerling, J. P.; Dijkhuizen, L. Isomalto/malto-polysaccharide, a novel soluble dietary fiber made via enzymatic conversion of starch. J. Agric. Food Chem. 2014, 62, 12034-12044

(21) Gangoiti, J.; Pijning, T.; Dijkhuizen, L. Biotechnological potential of novel glycoside hydrolase family 70 enzymes synthesizing $\alpha$-glucans from starch and sucrose. Biotechnol. Adv. 2018, 36, 196207.

(22) Gangoiti, J.; Corwin, S. F.; Lamothe, L. M.; Vafiadi, C.; Hamaker, B. R.; Dijkhuizen, L. Synthesis of novel $\alpha$-glucans with potential health benefits through controlled glucose release in the human gastrointestinal tract. Crit. Rev. Food Sci. Nutr. 2020, 60, 123146.

(23) Te Poele, E. M.; Corwin, S. G.; Hamaker, B. R.; Lamothe, L. M.; Vafiadi, C.; Dijkhuizen, L. Development of slowly digestible starch derived $\alpha$-glucans with 4,6- $\alpha$-glucanotransferase and branching sucrase enzymes. J. Agric. Food Chem. 2020, 68, 6664-6671.

(24) Vujičić-Žagar, A.; Pijning, T.; Kralj, S.; López, C. A.; Eeuwema, W.; Dijkhuizen, L.; Dijkstra, B. W. Crystal structure of a $117 \mathrm{kDa}$ 
glucansucrase fragment provides insight into evolution and product specificity of GH70 enzymes. Proc. Natl. Acad. Sci. U. S. A. 2010, 107, 21406-21411.

(25) Ito, K.; Ito, S.; Shimamura, T.; Weyand, S.; Kawarasaki, Y.; Misaka, T.; Abe, K.; Kobayashi, T.; Cameron, A. D.; Iwata, S. Crystal structure of glucansucrase from the dental caries pathogen Streptococcus mutans. J. Mol. Biol. 2011, 408, 177-186.

(26) Meng, X.; Pijning, T.; Dobruchowska, J. M.; Gerwig, G. J.; Dijkhuizen, L. Characterization of the functional roles of amino acid residues in acceptor-binding subsite +1 in the active site of the glucansucrase GTF180 from Lactobacillus reuteri 180. J. Biol. Chem. 2015, 290, 30131-30141.

(27) Waterhouse, A. M.; Procter, J. B.; Martin, D. M. A.; Clamp, M.; Barton, G. J. Jalview Version 2 - a multiple sequence alignment editor and analysis workbench. Bioinformatics 2009, 25, 1189-1191.

(28) Leemhuis, H.; Pijning, T.; Dobruchowska, J. M.; Van Leeuwen, S. S.; Kralj, S.; Dijkstra, B. W.; Dijkhuizen, L. Glucansucrases: threedimensional structures, reactions, mechanism, $\alpha$-glucan analysis and their implications in biotechnology and food applications. J. Biotechnol. 2013, 163, 250-272.

(29) Crooks, G. E.; Hon, G.; Chandonia, J. M.; Brenner, S. E. WebLogo: a sequence logo generator. Genome Res. 2004, 14, 11881190.

(30) Kumar, S.; Stecher, G.; Li, M.; Knyaz, C.; Tamura, K. MEGA $\mathrm{X}$ : Molecular evolutionary genetics analysis across computing platforms. Mol. Biol. Evol. 2018, 35, 1547-1549.

(31) Felsenstein, J. Confidence limits on phylogenies: an approach using the bootstrap. Evolution 1985, 39, 783-791.

(32) Jones, D. T.; Taylor, W. R.; Thornton, J. M. The rapid generation of mutation data matrices from protein sequences. Comput. Appl. Biosci. 1992, 8, 275-282.

(33) Dobruchowska, J. M.; Gerwig, G. J.; Kralj, S.; Grijpstra, P.; Leemhuis, H.; Dijkhuizen, L.; Kamerling, J. P. Structural characterization of linear isomalto-/malto-oligomer products synthesized by the novel GTFB 4,6- $\alpha$-glucanotransferase enzyme from Lactobacillus reuteri 121. Glycobiology 2012, 22, 517-528.

(34) van Leeuwen, S. S.; Kralj, S.; van Geel-Schutten, I. H.; Gerwig, G. J.; Dijkhuizen, L.; Kamerling, J. P. Structural analysis of the $\alpha$-Dglucan (EPS35-5) produced by the Lactobacillus reuteri strain $35-5$ glucansucrase GTFA enzyme. Carbohydr. Res. 2008, 343, 1251-1265. (35) Kabsch, W. XDS. Acta Crystallogr. D Biol. Crystallogr. 2010, 66, $125-132$.

(36) McCoy, A. J.; Grosse-Kunstleve, R. W.; Adams, P. D.; Winn, M. D.; Storoni, L. C.; Read, R. J. Phaser crystallographic software. J. Appl. Crystallogr. 2007, 40, 658-674.

(37) Murshudov, G. N.; Vagin, A. A.; Dodson, E. J. Refinement of macromolecular structures by the maximum-likelihood method. Acta Crystallogr. D Biol. Crystallogr. 1997, 53, 240-255.

(38) Emsley, P.; Lohkamp, B.; Scott, W. G.; Cowtan, K. Features and development of Coot. Acta Crystallogr. D Biol. Crystallogr. 2010, $66,486-501$.

(39) Joosten, R. P.; Long, F.; Murshudov, G. N.; Perrakis, A. The PDB_REDO server for macromolecular structure model optimization. $\bar{I} U C r J$ 2014, 1, 213-220.

(40) Pettersen, E. F.; Goddard, T. D.; Huang, C. C.; Meng, E. C.; Couch, G. S.; Croll, T. I.; Morris, J. H.; Ferrin, T. E. UCSF ChimeraX: Structure visualization for researchers, educators, and developers. Protein Sci. 2021, 30, 70-82.

(41) Kelley, L. A.; Mezulis, S.; Yates, C. M.; Wass, M. N.; Sternberg, M. J. E. The Phyre2 web portal for protein modeling, prediction and analysis. Nat. Protoc. 2015, 10, 845-858.

(42) Brzozowski, A. M.; Davies, G. J. Structure of the Aspergillus oryzae $\alpha$-amylase complexed with the inhibitor acarbose at $2.0 \AA$ resolution. Biochemistry 1997, 36, 10837-10845.

(43) Davies, G. J.; Brzozowski, A. M.; Dauter, Z.; Rasmussen, M. D.; Borchert, T. V.; Wilson, K. S. Structure of a Bacillus halmapalus family $13 \alpha$-amylase, BHA, in complex with an acarbose-derived nonasaccharide at 2.1 A resolution. Acta Crystallogr. D Biol. Crystallogr. 2005, 61, 190-193.
(44) Arnal, G.; Cockburn, D. W.; Brumer, H.; Koropatkin, N. M. Structural basis for the flexible recognition of $\alpha$-glucan substrates by Bacteroides thetaiotaomicron SusG. Protein Sci. 2018, 27, 1093-1101.

(45) Robert, X.; Gouet, P. Deciphering key features in protein structures with the new ENDscript server. Nucleic Acids Res. 2014, 42, W320-W324. 